\title{
Editorials
}

\section{Practice guidelines for necropsy: time for action}

The necropsy is a professional activity which requires extensive knowledge and technical ability in order to identify and interpret important findings within a wide range of clinical contexts. The central role of the medicolegal necropsy in the investigation of death necessitates the highest possible standards of practice. Similarly, the clinical necropsy represents one of the few situations when clinicians elect to submit their management of cases to assessment by other doctors. This issue of the Journal contains yet another study describing a high rate of discrepancies between clinical diagnoses and necropsy findings. ${ }^{1}$ Such studies are useful in that they demonstrate the amount of data that can be obtained from high quality necropsies. Pathology has been at the forefront of the introduction of quality control into medicine and it must surely be time for formal quality control in necropsy practice.

Practice guidelines represent recommendations that identify a range of voluntary strategies for the management of a specific problem and that also allow for practice variation due to individual circumstances. ${ }^{2}$ Standards differ from guidelines in that practice variation is not expected. Variation in necropsy practice is to be expected as each necropsy involves the incorporation of substantial patient specific information and consequently practice guidelines rather than standards are desirable and could be achievable if formulated through the framework of the appropriate professional pathology organisations..$^{2-4}$

\section{The necropsy examination}

Patient notes and consent forms should be studied carefully, particularly in relation to clinical problems and possible limitations placed on the examination by relatives. After identification and a careful external examination, the body is opened by a pathologist who should also remove the internal organs whenever practicable. The standard ' $\mathrm{Y}$ ' incision is recommended for the basic examination of both sexes. ${ }^{5}$ This incision minimises visible evidence of necropsy and permits easy access to the neck structures. The internal organs may be removed individually (Rokitanski's method), together in a single block (Letulle's method) or in three main blocks (thoracic, abdominal and pelvic blocks). Organs often overlooked at necropsy include testes, breasts and intestines. Unless the examination requires alternative approaches to demonstrate specific pathological processes, the pelvic floor should be left intact. The internal female genitalia should be removed with a short length of vagina, leaving generous cuffs of vagina and rectum. Sites of recent surgery are best examined with the appropriate clinician present.

The precise order in which individual organs and systems are dissected is not important but effort should be made to demonstrate important pathological processes. This may require modification of normal dissection routines and delay of final dissection until the demonstration of necropsy findings. The frequency with which individual organs should be dissected and weighed is contentious. All major organs (heart, lungs, brain, liver, and kidneys) should be dissected in order to facilitate examination of the blood and lymphatic supply in addition to relations with adjacent structures. These organs should be separated and weighed, although other organ weights may often be necessary. Fixation of the intact brain followed by detailed neuropathological examination produces a higher detection rate of abnormalities but may be impracticable in many centres.

Awareness of how to obtain appropriate samples for biochemical, microbiological or toxicological analyses is important and pathologists should have a full repertoire of special dissection techniques to enable examination of unusual sites. ${ }^{5}$ Examples would include the cardiac conducting system, vertebral arteries, temporal bone, sinonasal block, orbital contents, cervical spine, and other musculo-skeletal sites. Specific consent should be obtained for any potentially disfiguring procedure.

\section{Necropsy histology}

The retention of material from medicolegal necropsies is problematic and practices must be agreed with local coroners. Limited resources prevent histology on all cases and the aim should be to extract the maximum amount of information in the most cost-effective manner. Necropsy costings should include a basic histology component which is reduced by producing composite blocks containing several tissues. Some centres sample a standardised selection of tissues from every necropsy. ${ }^{2}$ This facilitates retrospective studies, particularly when these data are linked in an appropriate database.

\section{The necropsy report}

Organ weights and internal and external macroscopic findings should be recorded contemporaneously on a simple proforma. This can also serve as a histology request form for laboratory use and can be retained permanently unlike cassette tapes which are used repeatedly. The final necropsy report should include basic demographic details, a brief clinical summary, descriptions of the external and internal examinations with reports of histology or other investigations, a summary of findings with appropriate pathology coding, and a concluding commentary. ${ }^{367}$ This commentary should reconcile the clinical problems with the pathological findings, both expected and unexpected. All reports should include a cause of death in the standard international form of the medical certificate of cause of death as required by the Office for National Statistics. A summary of findings should be issued to clinicians within 48 hours. Any conclusions may be modified by further investigations and outlined in the final report, which should be issued within two to three weeks (neuropathology reports will require four to six weeks for completion). Performance should be monitored by regular audits of necropsy report quality and production times. ${ }^{8}$

\section{Clinicopathological considerations}

Necropsy reports are just one component of necropsy based clinicopathological communication, which is an integral part of necropsy practice. ${ }^{6}$ This process begins with completion of the necropsy consent form which should outline the relevant clinical problems. Necropsy demonstrations and clinicopathological meetings provide opportunities to enhance interest and knowledge by 
linking basic pathological science with applied clinical situations. Such interactions are enhanced by the use of photography or still video imaging.

\section{Conclusions}

Historically, the medical profession has ensured the quality of medical care by controlling the credentials of medical practitioners. In recent years attention has turned towards the assessment of outcomes or performance measures. Although necropsy represents an opportunity to study outcomes, the non-random nature of necropsy populations, the failure to collect relevant data and the lack of quality assurance for necropsy examinations currently precludes any meaningful benefits. If this situation is to change then pathologists must first put their own house in order and introduce guidelines for necropsy practice.

ROGER D START Lecturer in Pathology SIMON S CROSS Senior Lecturer in Pathology
Department of Pathology,

Sheffield University Medical School,

Beech Hill Road,

Sheffield S10 2UL

e-mail: s.s.cross@sheffield.ac.uk

1 Robinson IR, Marley NJE. Which factors predict cases with unexpected clinical findings at necropsy? F Clin Pathol 1996;49:909-12.

2 Hutchins GM and the Autopsy Committee of the College of American Pathologists. Practice guidelines for autopsy pathology: autopsy performance. Arch Pathol Lab Med 1994;118:19-25.

3 Hutchins GM and the Autopsy Committee of the College of American Pathologists. Practice guidelines for autopsy pathology: autopsy reporting. Arch Pathol Lab Med 1995;119:123-30.

4 Powers JM and the Autopsy Committee of the College of American Pathologists. Practice guidelines for autopsy pathology: autopsy procedures for brain, spinal cord and neuromuscular system. Arch Pathol Lab Med 1995;119:777-83.

5 Cotton DWK, Cross SS. The hospital autopsy. Oxford: ButterworthHeinemann, 1993.

6 Royal College of Pathologists. Autopsy and audit. London: Royal College of Pathologists, 1991.

7 Royal College of Pathologists. Guidelines for post mortem reports. London: Royal College of Pathologists, 1993.

8 Young NA, Naryshkin S. An implementation plan for autopsy quality control and quality assurance. Arch Pathol Lab Med 1993;117:531-4.

\section{Proliferative activity in invasive breast carcinoma}

Determination of proliferative activity provides important diagnostic and prognostic information in many tumours, including invasive breast cancer. Histological grading of breast carcinoma (which includes mitotic count), is now not only widely accepted as providing robust prognostic information but also as showing acceptable reproducibility. However, if grading is not carried out diligently by histopathologists on well fixed samples, the prognostic information provided is likely to be of relatively poor quality and in such a setting a more objective system, and also a simple technique for the determination of the growth fraction of a tumour, has long been sought.

Thymidine and 5-bromodeoxyuridine labelling, S-phase fraction assessed by flow cytometry and determination of the proportion of the tumour showing immunostaining with the Ki-67 antibody on frozen sections all have disadvantages. Disaggregation of tumour cells or access to fresh or frozen tissue may be needed. Large studies of patients with long term follow up have therefore been difficult to validate using these techniques. More recently, antibodies which can be used to determine the proliferation index of a tumour on paraffin wax sections have been described including MIB-1, Ki-S1 and anti-proliferating cell nuclear antigen (PCNA). The former recognises the $\mathrm{Ki}-67$ antigen, which is present in all cells active in the growth cycle - that is, not in $G_{0}$ phase, in routinely processed paraffin wax sections with the use of antigen retrieval ${ }^{1}$ and seems to be the most suitable of these antibodies. The technique is simple (although it may be affected by the $\mathrm{pH}$ of the buffering solution used) and correlates well with $\mathrm{Ki}-67$ immunoreactivity on frozen specimens, as described by Querzoli et al in this issue. ${ }^{2}$ A strong correlation between MIB-1 expression and histological grade has been described previously. ${ }^{34}$ There is also some evidence that MIB-1 expression may be relatively resistant to a delay in fixation of up to eight hours, ${ }^{5}$ whereas mitoses are significantly less robust and notoriously difficult to identify in poorly fixed tumours. Thus, MIB-1 evaluation may be valuable in the determination of the proliferation index in tumours which have not been fixed immediately and in which grading is therefore sub-optimal.

The MIB-1 labelling index shows strong correlations with other biological and histopathological features of invasive breast carcinomas, and also with clinical outcome. ${ }^{24}$ The results of Querzoli et al in this issue, assessing the proportion MIB-1 immunoreactivity by image analysis confirm that correlations with tumour size and survival are seen, but also a correlation with $\mathrm{p} 53$ and c-erbB-2 expression and inverse correlations with both oestrogen and progesterone receptor status. ${ }^{2}$ A correlation with lymph node stage (although using the TNM staging system) is more unexpected and has not been seen by other authors. ${ }^{34}$

The major benefit of determining the percentage area showing immunoreactivity by image analysis is the objectivity of the technique, but it should not be taken for granted that this ensures good intra- and inter-observer reproducibility. Poor reproducibility of the cell proliferation index using image analysis has been reported-for example, only $56 \%$ agreement in $\mathrm{Ki}-67$ immunostaining by three observers was seen by Makkink-Nombrado et al. ${ }^{6}$ These authors found that the poor reproducibility seemed to be related to a bias in the "random" field selection. In addition, although direct light microscopic visualisation ensures that only malignant cells are included in the proliferation index by image analysis, this necessitates accurate interpretation of tissue sections by fully trained staff to ensure only tumour tissue is included in the analysis. Despite these difficulties, previous small series have shown a good correlation $(r=0.77)$ between the image analysis determination and light microscopic estimate of the percentage nuclear immunostaining with MIB-1. ${ }^{7}$ Semiquantitative light microscopic assessment of MIB-1 immunoreactivity may be of use in examining proliferation in imperfectly fixed tumours

Whichever technique is selected for determination of the proliferation index of a tumour, it is imperative that strict criteria must be agreed and adhered to, whether in defining the characteristics of a mitotic figure or in ensuring the 